\title{
Research on Problems and Countermeasures of the Construction of Yunnan Natural Medicine Raw Material Base
}

\author{
Jianling QI, Yingchun LU*, Shengchao YANG, Junwen CHEN, Guanghui ZHANG, Guangqiang LONG \\ Yunnan Agricultural University \\ Kunming 650201
}

\begin{abstract}
Yunnan is an important raw material base for natural medicine in China. And natural medicine industry has become an important support for characteristic agriculture industry in Yunnan plateau and an important industry for increasing farmers' income and getting rich. This paper deeply studied the current situation of the construction of natural medicine raw material base in Yunnan, and put forward the specific development countermeasures in view of the problems existing in the construction of raw material base in Yunnan Province, such as the lag of new food source certification, the blindness of base scale development and the inadequate exploitation of core competitiveness of product. The purpose of this paper is to point out the direction for the transformation and upgrading of natural medicine raw material base construction in Yunnan Province, and to provide a reference for other regions in China to develop natural medicine base construction.
\end{abstract}

Keywords-Yunnan; Natural medicines; Raw material base; Chinese herbal medicine

\section{INTRODUCTION}

With the increasing call for the world returning to nature, natural medicines, health care products, foods \& drinks, dietary supplement, special medical products, daily chemical product, biopesticide, feed additives and so on made from natural medicine raw materials are becoming more and more popular. The international trade volume of botanical drugs has been growing rapidly, and the import \& export trade share of Chinese traditional medicine has been increasing continuously. China has put forward the development strategy of building a large health industry, and the large health industry with natural medicines as raw materials is a strategic emerging industry that our country focuses on building. The demand for raw materials has increased dramatically, so natural medicine raw materials industry has broad prospects for development.

Yunnan province has the most abundant natural medicine raw materials and health product resources in China. The industry of natural medicine raw materials, such as traditional Chinese medicine, medicine $\&$ food, health products and so on are the dominant characteristic biological industry for the development of regional economy in Yunnan province, and it is also the basic industry of Yunnan's biopharmaceutical large health industry. Driven by the provincial party committee and the provincial government, Yunnan's bio-medicine industry has maintained steady and rapid growth since 2016. In 2017, the total industrial output value of the province's 215 drug manufacturing enterprises reached 46.5 billion yuan, there are 8 enterprises whose total output value exceeded 1 billion yuan. The planting area in Yunnan province has ranked first in China for two consecutive years. The planting area of traditional Chinese medicine in 2017 increased by $20 \%$ year-on-year compared with that in 2016, and Yunnan's year-on-year growth of $12.36 \%$ accounted for $9.56 \%$ of the national planting area. The agricultural output value increased at a high speed of $22.6 \%$, and it had became a resounding business card for biomedicine industry in Yunnan.. However, there are still many problems in industrial development at present, in summary, they are mainly "Many varieties with few brands; Much raw materials with little processing; Many legends with little science". In view of these problems, the output value of Yunnan's natural medicine raw materials industry is expected to exceed 100 billion yuan in the next five years through scientific development, , and to exceed 200 billion yuan by 2025, Yunnan will become the most distinctive natural medicine raw material base, and lay solid foundation for the development of bio-medicine industry, it has great significance in promoting regional economic development in Yunnan province, increasing farmers' income and maintaining frontier stability.

\section{DEVELOPMENT STATUS OF NATURAL PHARMACEUTICAL RAW MATERIAL BASE IN YUNNAN PROVINCE}

\section{A. The Construction of Traditional Chinese Medicine Base Has Been Fully Accelerated}

In recent years, the construction of traditional Chinese medicine industry base in Yunnan province has been promoted constantly, and the construction of traditional Chinese medicine planting (breeding) base has been fully accelerated First, since 2009, the province has identified 60 sites as the hometown of Yunnan medicine, 103 seed-breeding base of traditional Chinese medicine, 144 traditional Chinese medicine planting (breeding) science \& technology demonstration garden, and 25 traditional Chinese medicine processing science \& technology enterprises. More than 40 industry key counties(city or area)have sprung up with industry output value 
exceeding 100 million yuan, the production areas of traditional Chinese medicines have developed steadily, and the brand construction of "the hometown of Yunnan medicine" the authentic traditional Chinese medicine has achieved remarkable results. Second, in 2014, the agricultural output value of panax notoginseng reached 12.2 billion yuan driven by the raw material market of traditional Chinese medicine, and it has become a leading industry with an agricultural output value of over 10 billion yuan. A number of superior varieties of traditional Chinese medicine have sprung up, such as dendrobium, gastrodia elata and gentiana rigescen and so on, whose output value achievements may be close to 1 billion yuan. Among the large varieties of Chinese medicine with strong influence and large scale in the industry, the superiority is prominent. Third, in 2014, the planting area of traditional Chinese medicine in the province was about 2.61 million mu, achieving an agricultural output value of about 20 billion yuan. In the past five years, the planting area of traditional Chinese medicine in the province has been growing rapidly at an average annual rate of $12.8 \%$, and the agricultural output value has been growing at a high speed of $33.9 \%$, the industry development shows a "blowout" growth. The rapid development of industry in Yunnan province has become an important support for the large health industry of bio-medicine and a new highlight of regional economic development

\section{B. The Construction of Raw Material Base of Health Industry Develops Rapidly}

With the rapid development of large health industry in China, the rapidly expanding market demand has promoted the rapid development of raw material base construction of health products in Yunnan Province. First, for a batch of dual-use medicine \& food products, including amomum tsaoko, job's tears, sealwort, poria cocos, finger citron, honeysuckle, fructus mume, papaya, semen lablab album, pepper and so on, the rapid growth of the market demand for health product raw material promoted their rapid growth in the food field beyond the field of traditional Chinese medicine, the construction scale of planting base have been continuously extended, with a planting area of 1.82 million mu and the agricultural output value of 3.2 billion yuan. Second, the demand for health food raw materials such as amorphophllus, maca, Luoping little yellow ginger, wild pepper, marigold, spirulina platensis, edible rose, haematococcaceae, moringa oleifera and so on is exuberant. The planting scale of raw materials reached 1.64 million $\mathrm{mu}$, and the agricultural output value reached 6.4 billion yuan. The construction of raw material base for health products was accelerated, a number of leading industry enterprises and product brands were gradually formed, and industrial development entered an accelerating period.

\section{The Quality Standard System is Basically Formed}

First, 370 standards for traditional Yi medicine and Dai medicines and local traditional Chinese medicine were formulated and revised, the standard system of ethnomedicines, traditional Chinese medicine and decoction pieces of traditional Chinese medicine has been improved continuously, laying a good foundation for industry development. Second, the establishment of product quality standards of a number of geographical indications[1], such as Wenshan notoginseng,
Zhaotong gastrodia elata, Honghe erigeron breviscapus, Eryuan plum, Chenghai spirulina platensis, Lum dendrobium, Longling purple dendrobium and so on, and a number of local standards, such as Wenshan notoginseng, dendrobium officinale and so on. Third, 12 GAP base was established for 8 kinds of superior traditional Chinese medicine, including notoginseng, erigeron breviscapus, saussurea lappa, dendrobium officinale, P. polyphylla var. Yunnanensis, spirulina platensis, angelica sinensis, periplaneta americana and so on. Fourth, more than 40 new varieties of plants for natural medicine raw material, such as notoginseng, erigeron breviscapus, amorphophllus, P. polyphylla var. yunnanensis, sealwort, common bletilla pseudobulb, finger citron, Yunnan angelica sinensis, saussurea lappa, gentiana rigescen and so on, has been selected and planted, and the DUS standards for natural medicine materials such as notoginseng, erigeron breviscapus, marigold and so on has been established, the building process of system for variety of natural medicine raw material is accelerated, the quality standard system of traditional Chinese medicine is basically formed, this has laid a solid foundation for the further development of the industry.

\section{Leading Enterprises and Brand Influence have been Significantly Improved}

First, relying on the resources and industrial advantages of natural pharmaceutical raw materials, industrial enterprises have gathered together, there are more than 1,000 natural pharmaceutical raw materials or involving industry enterprises and professional cooperation organizations, industry leading enterprises such as Yunnan Baiyao, Kunming Pharmaceutical, Miao County notoginseng, Bogao Biotech, Takata notoginseng, Guangming dendrobium, Spirin and so on have sprung up. Second, with the promotion of natural pharmaceutical raw material, such as Herbs and extracts of notoginseng, dendrobium officinale, breviscapinun, lutein, spirulina platensis, astaxanthin and so on, the industrial base has been expanded and the brand awareness of natural pharmaceutical raw material has been improved. Third, the influence of Yunnan natural pharmaceutical raw material industry has been significantly enhanced through the identification and construction of "the hometown of Yunnan medicine", traditional Chinese medicine industrial park, sciencetechnology demonstration park and traditional Chinese medicine breeding base.

\section{E. The Supporting Capacity of Science and Technology has been Significantly Enhanced}

First, a number of national and provincial research and development platform, such as the state \& local engineering research center for new product development of the southwest ethnic medicine, notoginseng resources and health engineering research center of Yunnan Province, notoginseng engineering technology research center of Yunnan Province, research center of standardized planting of superior traditional Chinese medicine in Yunnan Province, erigeron breviscapus GAP engineering technology research centre of Yunnan Province, as well as a batch of enterprise technology center and academician experts workstation, have been established. Second, a number of key industry technology on germplasm innovation, standardized planting and quality control of key 
natural pharmaceutical raw material have been constantly worked out, the outputting of achievements in natural pharmaceutical raw material, such as new variety, patent, reward are accelerated, "Field investigation and development of natural medicine resources in low latitude plateau region (Yunnan)" won the first prize of national science and technology progress, "Chemical study of schisandra fruit" won the special prize of natural science in Yunnan Province, academician Jun ZHOU and Handong SUN, who are engaged in research on the utilization of natural pharmaceutical raw material, won the outstanding contribution award of Yunnan Province. Third, through constantly strengthening the construction of industry support system and relying on the project of office of the rural comprehensive reform leading group under the state council, the socialized service system of traditional Chinese medicine in Yunnan province was preliminarily constructed, the achievement transformation and industrialization were accelerated, and more than 180 scientific and technological achievements were transformed, and remarkable economic benefits has been obtained.

\section{EXISTING PROBLEMS}

In recent years, although the construction of natural pharmaceutical raw material base in Yunnan province has achieved rapid development, there are still some urgent problems need to be solved.

\section{A. Lag of New Food Source Certification.}

In the natural medicine raw material industry, the development of traditional Chinese medicine industry is becoming more and more mature due to the strong pull of the demand for raw materials of traditional Chinese medicine. However, the market expansion of traditional Chinese medicine has been severely limited, as the large varieties of traditional Chinese medicine, such as notoginseng, erigeron breviscapus, dendrobium officinale, gastrodia elata and so on, have not got new food material certification, the development of the industry of dual-use medicine $\&$ food and health products is still in its infancy, and it is urgent to accelerate the development of the industry of dual-use medicine $\&$ food and health products raw materials.

\section{B. Great Blindness in Base Scale Development}

The construction scale of industrial base for natural pharmaceutical raw material such as notoginseng, dendrobium, maca and so on, increased too quickly, the relationship between product supply and demand is seriously out of balance, the " roller - coaster" development pattern of part of the raw material industry needs to be changed.

\section{Lack of Core Competitiveness of Products}

Yunnan's good ecology and rich ethnic culture connotation are the advantages of natural medicine development in Yunnan. However, due to the fact that the production organism of raw drug raw materials is not high enough, the industry supervision is difficult. The production mode aiming at the output of the industry has made some natural medicinal materials such as notoginseng, Dendrobium candidum and other agricultural residues and heavy metals exceed the standard prominently What's more, the original ecology and rich national culture connotation of production area of "Yunnan medicine" are not fully explored, and the product market competitiveness and influence still need to be improved.

\section{Weak Market Development Ability of Leading Enterprises}

Yunnan's leading enterprises in the production and marketing of raw materials for natural medicines are constantly forming and expanding in scale, but overall speaking, the leading enterprises are small in scale and weak in market development ability. The fact that the leading effect of professional cooperative organizations is not obvious has not been changed.

\section{E. Lack of Scientific and Technological Talents}

There is a lack of industrial talents, lagging behind in basic research, insufficient driving force of industrial innovation, imperfect system of transformation of scientific and technological achievements and promotion and service, low efficiency of transformation of achievements, and difficult to meet farmers' technological needs.

\section{F. Lack of Awareness for External Exchange}

With the implementing of "The Belt and Road" strategy in China, the advantages of Yunnan's radiation to South Asia and Southeast Asia are gradually emerging. However, there are few international exchanges and cooperation of natural pharmaceutical raw material of Yunnan Province, especially the promotion of strategic positioning towards the radiation center in Southeast Asia and South Asia is obviously inadequate.

\section{G. Management and Promotion Mechanisms Need to be Optimized}

The construction of natural pharmaceutical raw material base involves a large number of provincial departments, the status of the provincial science and technology department as an industry leading department is still unclear, some difficulties in the coordination among departments, industrial management and promotion mechanisms need to be strengthened.

\section{STRATEGIES TO ACCELERATE THE SOUND DEVElopment of YunNan Natural MEdicine Base}

\section{A. Upgrade the Raw Material Base of Traditional Chinese Medicine}

Focusing on large varieties of natural medicinal materials such as notoginseng, rhizoma paridis, dendrobium, gastrodin, erigeron breviscapus and so on, sticking to the development principle of appropriate scale, high level, remarkable benefit and intensive agglomeration taking the market demand as the guide and according to the modern agricultural mode of "breeded germplasm, standardized cultivation, digitized quality, industry in base" with ecological development path, it is necessary to update the technology existing in Chinese large medicine variety cultivation (breeding) bases Driven by the demand for new food raw material, the new food material 
certification of notoginseng stem and leaf, fresh strip of dendrobium officinale, gastrodia elata and so on shall be accelerated, the new food material certification of notoginseng earthnut, erigeron breviscapus, purple dendrobium, drumstick dendrobium flower and so on shall be started, the health product area of traditional Chinese medicine shall be enlarged, traditional Chinese medicine GAP base for sealwort, bletilla striata, fingered citron, rhizoma paridis, gallbladder and so on shall be built, so as to promote the standardization and scale development of industry. The industrialization of Dai medicine and Yi medicine shall be promoted, ethnomedicine resources such as Yunnan Tibetan medicine, Haney medicine, Lexical medicine and so on shall be excavated and protected, ethnomedicine quality standards of beaks aconitum, gingershape notoginseng, wild notoginseng and so on should be established to promote the protection and application of ethnomedicine resource. Combining with the fourth national general survey of Chinese medicinal resources, the study on the protection and utilization of wild and endangered Chinese medicinal resources should be carried out, and it should establish Aconitum yunnanensis, Swertia militaris and other introduction, domestication and cultivation bases to promote the original habitat protection of wild Chinese medicine resources. It is necessary to step up scientific and technological cooperation with bases of natural medicinal materials in Southeast Asia and South Asia, establish large databases of medicinal plants, and promote the protection and sustainable use of medicinal plant resources abroad.

\section{B. Expand the Base of Raw Materials of Healthy Food}

Based on the rich and colorful natural pharmaceutical raw material in Yunnan and complying with the urgent need of society for the development of great health products, the great health product territory is enlarged around the market requirement of natural health product. The new product exploitation level of job's tears, edible roses, spirulina platensis, haematococcaceae, wild pepper, maca, amomum tsao-ko, moringa oleifera, sealwort, marigold, Luoping little yellow ginger, poria cocos, fingered citron, honeysuckle and so on in the area of food, health care products, cosmetics, special medical products, feed additives, biopesticide and so on. The industrial quality standards and production technical procedures shall be improved and upgraded, the effect interpretation of raw material and great health experience shall be explored, so as to enlarge the demands for raw material of great health products. Focused on the industry layout, health food industry park shall be established and the construction of industry base for great health product shall be accelerated.

\section{Create Industry Leading Enterprise Group}

It is necessary to optimize the layout of the natural pharmaceutical raw material industry and guide enterprises to gather in the industrial core areas, industrial development areas and industrial growth poles. First, create a favorable investment and development environment, encourage domestic and foreign pharmaceutical enterprises to invest in Yunnan to establish the raw material industry of Chinese medicines, and attract domestic and foreign enterprises, research institutes, universities and various social forces to invest in the raw material industry of Yunnan's natural medicines in various forms. Second, support the establishment and development of leading enterprises and professional cooperative organizations in the natural pharmaceutical raw materials industry, encourage professional cooperative organizations to upgrade to the leading enterprises, and foster local enterprises groups in the natural pharmaceutical raw materials industry. Third, support enterprises as the main body, carry out industrial technological innovation, guide the integration and reorganization of industrial enterprises, and promote the development of scale, modernization, internationalization and branding Fourthly, guide the leading enterprises in the base of natural medicines and raw materials to make technological innovations, increase investment in research and development, strengthen the technological research centers of national and provincial enterprises, high-tech enterprises, provincial innovative pilot enterprises, innovative platforms, innovative talents and innovative teams, cultivate innovative leading enterprises, and accelerate the implementation of innovation driven development strategy. Fifth, support enterprises to engage in the production of organic, green and ecological raw materials for natural medicines, participate in park construction, gather and develop, and form scale effects; guide enterprises to strengthen scientific and trade exchanges and cooperation with domestic and foreign enterprises, carry out various forms of publicity activities, and create a good industrial atmosphere for the development of enterprises. We should cultivate key leading enterprise clusters through "raising stocks, expanding fields and expanding increments".

\section{Enhance Market Competitiveness of Product}

We will establish consumer demand oriented production mode of product of natural pharmaceutical raw material in Yunnan, establish large data center of healthy industry in Yunnan through increasing the publicity of different media, and realize the base online, production online, testing online and research online, so as to provide whole process data support for the sale of product of natural pharmaceutical raw material, and create the brand of pure natural and original ecology product of natural pharmaceutical raw material in Yunnan. We will enhance consumers' confidence in products and competitiveness of products, annotate and verify product functions, develop new products and carry out large health experience through the implementation of Yunnan natural medicine raw material large healthy product experience program. Based on the rich Yunnan medicine culture and ethnomedicine culture, the cultural connotation of natural pharmaceutical raw material in Yunnan is deeply explored to provide cultural support for the establishment of Yunnan medicine brand. We will accelerate the construction of thirdparty testing platform for natural pharmaceutical raw material in Yunnan, carry out product quality evaluation through industry cooperation organizations such as the industry technical association and the industrial alliance, and strengthen market supervision, so as to guarantee the brand building of products of natural pharmaceutical raw material in Yunnan. 


\section{E. Strengthen the Driving Force of Scientific and Technological Innovation}

Focusing on the core area of Kunming, we will improve the driving ability of innovation in natural pharmaceutical raw material, overcome a series of key technical problems that restrict the development of natural pharmaceutical raw material industry, and drive the development of natural pharmaceutical raw material industry in Yunnan. Focused on key varieties such as notoginseng, dendrobium, rhizoma paridis, medicine resources in South China and so on, the research and development capabilities of national and provincial $R \& D$ platforms will be integrated and enhanced, and a number of provincial R\&D platforms will be built, enterprises are supported to establish and improve technology centers, basiclevel academician expert workstations will be established, and industry technology innovation and achievement transformation will be promoted. We will carry out the "Yunnan medicine genome project", and complete the whole genome sequencing of 20 key medicinal plants such as notoginseng, erigeron breviscapus, dendrobium officinale, maca and so on, conduct a census of traditional Chinese medicine resources, and protect rare and endangered traditional Chinese medicine resources. A number of key technologies for notoginseng, erigeron breviscapus, gastrodia elata, dendrobium, rhizoma paridis and so on has been overcome, such as whole gene selection breeding, successive cropping obstacle, native habitat planting, ecological prevention and control of diseases and pests, quality control, gene-exploration, biosynthesis of key active ingredients and so on, and the industry technology upgrading will be promoted. We will formulate the European Union or the United States pharmacopoeia standards for important traditional Chinese medicine such as notoginseng, erigeron breviscapus and so on, and support the establishment of quality standards for geographical indication product certification and new food raw material certification, and establish the quality standard system for traditional Chinese medicine. We will improve the technical service system of natural pharmaceutical raw material industry in Yunnan, and strengthen the conversion and promotion of technological achievements, as well as improve the technical level of natural pharmaceutical raw material industry. We will guide relevant units to actively carry out scientific and technological cooperation and exchanges focused on southeast Asia and South Asia, train and introduce high-end technology and industry talents, academic and technological leaders, technological innovation talents and innovation teams, enhance the capacity of scientific and technological innovation, and enhance the core competitiveness of the industry as well.

\section{F. Accelerate the Certification of New Food Material}

We will focus on the new food material certification for Yunnan notoginseng, erigeron breviscapus, purple dendrobium, drumstick dendrobium flower and so on. The safety assessment and new food material certification aimed at basic new food material such as notoginseng earthnut, erigeron breviscapus, purple dendrobium, drumstick dendrobium flower and so on that are edible for Yunnan folk should be carried out, it will provide support for the expansion of health product raw material of traditional Chinese medicine.

In conclusion, the construction of Yunnan natural medicine base should give priority to ecological development, highlight local advantages and special varieties, expand the whole industry chain, constantly improve the scale, organization and scientific level of the natural medicine industry, and continuously develop natural medicine on the basis of meeting the needs of traditional Chinese medicine for safe, healthy and effective products. In the field of development, we should steadily promote the application of natural medicines in health products and food, and make Yunnan's natural medicines industry bigger and stronger.

\section{ACKNOWLEDGMENT}

Jianling QI, male, associate professor of College of Economics \& Management of Yunnan Agricultural University, postgraduate tutor, Major research direction industrialization of traditional Chinese medicine.

E-mail: qijianling@163.com.

Corresponding author: Yingchun LU, female, State assets management office of Yunnan Agricultural University, Major research direction cultivation of traditional Chinese medicine.

E-mail: 351545297@QQ.com.

\section{REFERENCES}

[1] Jianling QI, Industrial development research of Longling dendrobium Journal of Yunnan Agricultural University, 2013,7 (4): 1-5.

[2] Feng LU, Problems and strategies for the problems in the processing of traditional Chinese medicine[D]Northwest Agriculture \& Forestry University, 2011. 